\title{
Container Cap Type Code
}

National Cancer Institute

\section{Source}

National Cancer Institute. Container Cap Type Code. NCI Thesaurus. Code C93541.

A coded value specifying the type of container cap. 